\title{
Optimal Placement of PMU of the Iraq Network for Full Observability Assessment
}

\author{
Qasim Kamil Mohsin, Xiangning Lin, Asad Waqar, and Samir Mohammed
}

\begin{abstract}
To better performance and real time observability to Iraq networks $(400 \mathrm{KV}, 132 \mathrm{KV})$ by setting up PMU in different sites of the networks. In this paper the Power system observability analysis and the rules with phasor measurement unit (PMU) are presented. it is unnecessary as well as uneconomical to status PMU in each bus, therefore there are various algorithms: Depth First Search, a graph-theoretic procedure, Annealing Method, Rec. spanning Tree, Direct spanning Tree, Dir (N-1) Spanning Tree, Binary integer programming and Linear programming, are applied to find optimal number and optimal placement of PMU to obtain fully observability of the system in two cases: first case each bus can be seen at least one time from PMU and second case each bus can be seen at least two times from PMU, to networks of systems: IEEE 9-bus, IEEE 14-bus, IEEE 24-bus. Simulation results proved amongst optimal solutions obtained from these algorithms, it be by use binary integer programming therefore, applied it to obtained optimal number and optimal placement of PMU to Iraq networks $(400 \mathrm{KV}, 132 \mathrm{KV})$ to observability whole system for these two cases.
\end{abstract}

Index Terms-Binary integer programming, observability, optimal PMU placement (OPP), PMU.

\section{INTRODUCTION}

Highlight Iraq's transmission network consists of 400KV and $132 \mathrm{KV}$ systems; the entire system consists of around 25 substations at $400 \mathrm{KV}, 233$ substations at $132 \mathrm{KV}$. The $400 \mathrm{KV}$ grid transmission systems was introduced in the 1970 s due to the inability of the $132 \mathrm{KV}$ network to handle the increase in load demand as well as This system acts as reliable way transmitting power from the South and North regions to the central region. During the recent years, new generation stations, substations and some transmission lines have been constructed across the different situation of country [1]. All efforts put to increase the amount of power generation to handle demand of the loads but not to improvement performance of the system. The Iraq transmission networks operate with large capacity and a high complexity, the observability of the whole system it can be done by The National control center which consist from three regional control centers; north, middle, and south. Theses regional control center obtained the information about substations related to its regional places.

The reliability of the system is a measure of the system ability to provide an electrical energy consistently considering two basic aspects: adequacy and security.

Manuscript received July 2, 2015; revised August 25, 2015.

The authors are with the State Key Laboratory of Electromagnetic Engineering, Huazhong University of Science and Technology, Wuhan 430074, Hubei Province, China (e-mail: phd.qasim@yahoo.com)
Adequacy relates to the existence of sufficient generation with transmission and distribution facilities to supply requirements of the loads at all times. Security, relates to the ability of the power system to withstand on local and widespread disturbances [2], [3].The Security and reliability operations of power systems networks required close monitoring of the system operating conditions. This is accomplished traditionally by state estimator which exists in the control centre computer and has access to Reliable the received measurements from numerous substations. These measurements are commonly provided by the remote terminal units (RTU) at the substations and include power injections, active/reactive power flows, and bus voltages and branch currents magnitudes, circuit breaker state (on/off), and tap changer position of transformer [4]. The reliability of the system observability by using the conventional supervisory control and data acquisition (SCADA) measurements it is limited, because low sampling rate and relatively low accuracy of measurements [5]. The Recent years shows that the minor instability in one part of the network can lead to failure of large parts of the system. For this and many other reasons this paper, urges efforts to utilizing advanced computing networking and new modernized and intelligent, measurement technologies. It will enable usage efficient energy, and increased reliability of the system. One of those new technologies is a utilizing modern measurement device known as a Phasor Measurement Unit (PMU), devices, which are used synchronization signals from the global positioning systems (GPS) satellites and provide measures voltage and current phasor in a power system with higher accuracy which be able to watching the entire system state in real time. The reduce effects of scale time delay among measurements, useful for many other applications such as system control, protection, stability assessment, and state estimation accuracy improvement [4]. Reference [6] shows, the important factors to preserving power system security through estimation situation awareness with accuracy of measurements in real time govern the formation in power system control center. The numbers of PMU installed in different locations of a power system network provides better information about numerous electricity quantities e.g. phasors currents, voltages, active and reactive power, all of them based on the same GPS reference time, whereby allows fast and reliable emergency actions, In order to avoid the worst contingency case by remedial measures implemented by new functions various based to phasor measurement [4]. In recent years the power systems network serviceable connect each other on large scale, it is unnecessary as well as uneconomical to place one PMU in each bus bar. Therefore the optimal PMU 
placement (OPP) problem formulated as the whole network observability by using small number of PMUs relatively much less than the number of buses in the system [7], [8].

Several methods for PMU placement problem developed in the literature considering network observability, integer programming (IP) approach in [9], [10]. In [11] the Tabu search met heuristic method is proposed to obtain minimum number of PMU for system observability. Optimal PMU placement to state estimation of Buses with zero and nonzero injections, and branches with power flow measurements use integer programming developed in [12]. Optimal PMU placement with available SCADA measurements with considering single and multiple measurement loss is reported in [13]. Binary particle swarm optimization (BPSO) is proposed in [14]. Depends on the reliability of PMUs, Multi objective function based genetic algorithm approach in [15], [16].

The approaches used in this paper are numerical and topological observability. Numerical observability it used the connectivity matrix or the Jacobean measurement, which reflect the system configuration. Topological observability method based ensures full observability, but do not ensure full ranked of connectivity Matrix measurement. The monitoring of certain grid events like generation unit failure or transmission line failure must be possible from the selected PMU locations. In addition, PMU must be distributed evenly in order to observable the critical parts of the network. The objective work in this paper is to find the minimum number of PMUs to make system wholly observable, as well as the optimal placements of these PMUs. The OPP methodologies applied include the system observability during normal operating conditions, as well as during single PMU outages. The schemes are discussed and compared to obtain OPP solutions to full observability of the system with minimum number of PMU and maximum redundancy are applied to IEEE 9-bus, IEEE 14-bus, IEEE 24-bus test systems, Iraq network $132 \mathrm{KV}, 400 \mathrm{KV}$.

This paper organized as follows: Section II describes Optimal PMU placement of power system observability, principle and rules. Section III Offers base of study illustrating Optimal PMU Placement principle, possibility of loss or failure of PMUs is considered in that study. Section IV is simulation with results and discussions of optimal number and optimal placement are analyzed with different algorithms. Conclusions are drawn in based on simulation results in Section V.

\section{PMU OF POWER SYSTEM OBSERVABILITY ANALYSIS AND RULES}

\section{A. Power System Observability Analysis}

Observability of the Power system is precondition of state estimation at real time and monitoring of the system under normal and abnormal conditions. There are two types to Observability of the node, one it's a directly observable node where a PMU is placed to measurements electrical/unelectrical quantities on it directly. Another node called indirect Observability node where is the measurements electrical quantities on it indirectly (calculable), there it is a connection to at least one another node supplied with PMU. A node is unobservable when missing one or more variables needed to determine its condition. The network is said to be complete observable if the measurements are sufficient to observable, states of all the nodes of the system are directly/indirectly observable, otherwise where some nodes are unobservable, the network is said to be incomplete observability [17]. The observability of Power system analysis can be done mainly in two different ways, namely, numerical and topological observability methods [18]. In both methods required to know number of buses $(\mathrm{N})$ and branches which interconnecting of these buses of the system. After known these parameters then it can be find optimal PMU number and placement. In The Numerical observability methods use of the connectivity matrix of a system, which obtained from the branches between buses to checks whether the rank of connectivity matrix is full observability or not. The representative of the numerical algorithms are integer linear programming(ILP), binary integer programming (BIP) which are capable to solving large-scale of problems in a short time and achieving optimal solution. IN Topological observability analysis methods are based on topological graph; the graph has ' $\mathrm{N}$ ' number of nodes representing the network buses and branches of the network which interconnecting of these buses. In topological approach the optimal PMU Placement set is searched such that each bus of the network is observable by at least one PMU. The representative graphical algorithms are Depth First Search, a graph-theoretic procedure, Annealing Method, Rec. spanning Tree, Direct spanning Tree, and Dir (N-1) Spanning Tree. Achieving The Topological Observability uses ILP concepts to find the locations for the PMU placement to make the system completely observable. The rules for PMU based topological observability are given as follows.

\section{B. PMU Placement Rules}

A PMU is able to measure the voltage phasors of the installed bus and the current phasors of some or all the lines connected to that bus. To make the system observable using the following rules can be used for PMU placement, have been applied:

Rule 1: For PMU installed at a bus, the bus voltages and the current phasors of all incident branches are known.

Rule 2: If voltage phasors and current phasors at one end of a branch are known, voltage phasors at the other end of that branch can be obtained using Ohm's law.

Rule 3: If voltage phasors at both ends of a branch are known, the current phasors through this branch can be calculated. The measurements such as bus voltage phasors and branch current phasors, directly obtained from PMUs, employing rule 1 are referred to as direct measurements; measurements derived by employing rule 2 and rule 3 are referred to as indirect measurements, or pseudo measurements as in. When the voltage phasors at a bus can be obtained either from direct measurements or indirect measurements, this bus is identified as observable. In an observable network, every bus must be observed at least once by using direct or indirect measurements [19]. The pseudo-measurement means calculate the require measurement indirectly by the KCL, KVL, using the 
Correlative measurements. It can improve the convergence of the result by applying these rules in a variety of algorithms, so that it can reduce the number of the PMUs. The objective of the optimal PMU placement (OPP) problem is the strategic choice of the minimum number of PMUs and the optimal location of the PMUs in order to ensure complete observability and satisfy a preset redundancy. After place a new PMU, whatever method is used, the observability of power system must be checked. If the system is observable, then the placement stops, else the placement must be continued for the random inputs until ascertain the state of power system is observability [7].

\section{PMU PLACEMENT FORMULATION}

\section{A. Optimal PMU Placement Principle}

A PMU placed at bus will measure the voltage phasor and currents phasors of outgoing branches of that bus. The number of the measured current phasors depends on the number of PMU channels availably. it is assumed that a PMU placed at bus will measure all current phasors of the branches connected to that bus, in addition to the voltage phasor of bus. Therefore, with the absence of any conventional measurements in the system, bus will be observable if at least one PMU is placed within the set formed by bus and all buses incident to it. Therefore, the objective of optimal PMU placement problem is to determine the minimum number of PMUs so as to preserve the system observability [20].

The Optimal number and optimal placement of PMUs to observability of the whole system, an important problem to be solved in power system. the OPP problem formulated as binary integer linear equations, by which the binary decision take variables $(0,1)$ to determine whether install PMU or not at buses, while preserving the whole network observability by using minimum number of PMUs.

The PMU placement at a bus can be seen as a binary decision variable defined as [18]-[20]:

$$
x_{i}=\left\{\begin{array}{l}
1 \text { if PMU is placed at bus } i \\
0 \text { Otherwise }
\end{array}\right.
$$

The optimal PMU placement problem for the system with $\mathrm{n}$ buses can be formulated as an integer linear equations problem as follows:

$$
\min F=\sum_{i=1}^{n} c_{i} x_{i}
$$

Subject to observability constraints:

$$
f_{i}=\sum_{j=1}^{n} a_{i j} x_{i} \geq 1 ? \quad i=\ldots \ldots . n
$$

where: $x i$ is a binary decision variable associated with bus $i$, $c_{i}$ is cost of installing a PMU at bus $i$, and assumed to be one per unit, $f_{i}$ represent number of times that $i$ th bus is observed through PMU measurements, $a_{i j}$ is the $i-j$ th entry of network connectivity matrix defined as :

$$
a_{i j}=\left\{\begin{array}{l}
1 \quad \text { if } i=j \text { or if } i \text { and } j \text { are connected } \\
0 \quad \text { Otherwise }
\end{array}\right.
$$

After getting the optimal number of PMUs, expression for redundancy $(\mathrm{R})$ measurement is given as:

$$
R=\sum_{k=1}^{p} A \cdot L(k)
$$

where, $R$ is redundancy of system, $p$ is the total optimal number of PMUs, A is the connectivity matrix, and L is the location of PMUs at bus (k). Eq.(5) gives the redundancy measurement for all the possible results of optimal location of PMUs. To illustrate the relations above take the example of 7-bus system is used as shown in Fig. 1, the numbers in the Fig. 1 represents bus number and lines between them represent transmission lines which interconnections these buses.

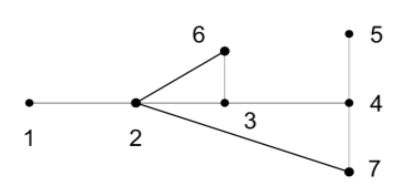

Fig. 1. System with 7- bus.

By rewriting the objective function of Eq.(2) to obtained Eq.(6)

$$
O . F=\min 2 x_{1}+x_{2}+\quad+x_{7}
$$

The observability constraints of Eq. (3) are listed as follows in Eq. (7):

$$
f(x)=\left\{\begin{array}{lc}
f_{1}=x_{1}+x_{2} & \geq 1 \\
f_{2}=x_{1}+x_{2}+x_{3}+x_{6}+x_{7} & \geq 1 \\
f_{3}=x_{2}+x_{3}+x_{4}+x_{6} & \geq 1 \\
f_{4}=x_{3}+x_{4}+x_{5}+x_{7} ? & \geq 1 \\
f_{5}=x_{4}+x_{5} & \geq 1 \\
f_{6}=x_{2}+x_{3}+x_{6} & \geq 1 ? \\
f_{7}=x_{2}+x_{4}+x_{7} & \geq 1
\end{array} ?\right.
$$

The objective function in (6) is the total number of PMUs required for complete system observability, which has to be minimized. the observability constraints in (7), The operator "+" serves as the logical "OR" and the use of 1 in the right hand side of the inequality constraints ensures that at least one of the variables appearing in the sum will be non-zero. The constraint $f_{1} \geq 1$ implies that at least one PMU must be placed at either one of buses 1 or 2 (or both) in order to make bus 1 observable. Similarly, the second constraint $f_{2} \geq 1$ indicates that at least one PMU should be installed at any one of the buses $1,2,3,6$, or 7 in order to make bus 2 observable and same away for others buses.

Solution of problem in (6), (7) shows that for full system observability, a minimum of two PMUs are required at busses 2 and 4 or at buses 2 and 5 and since bus 4 more branches than bus 5, so it can be more redundancy measurements . As a matter of fact, the set of busses where the PMUs have to be installed correspond to a dominating set of the network. Therefore, minimum PMU placement problem maps to smallest dominating set problem on the network. The zero injection buses and conventional measurements don't take in this paper to observability of critical part of grid. 
TABLE I: OPTIMAL NUMBER AND PLACEMENT OF PMU (OBSERVABILITY EACH BUS ONCE) FOR DIFFERENT ALGORITHM METHODS

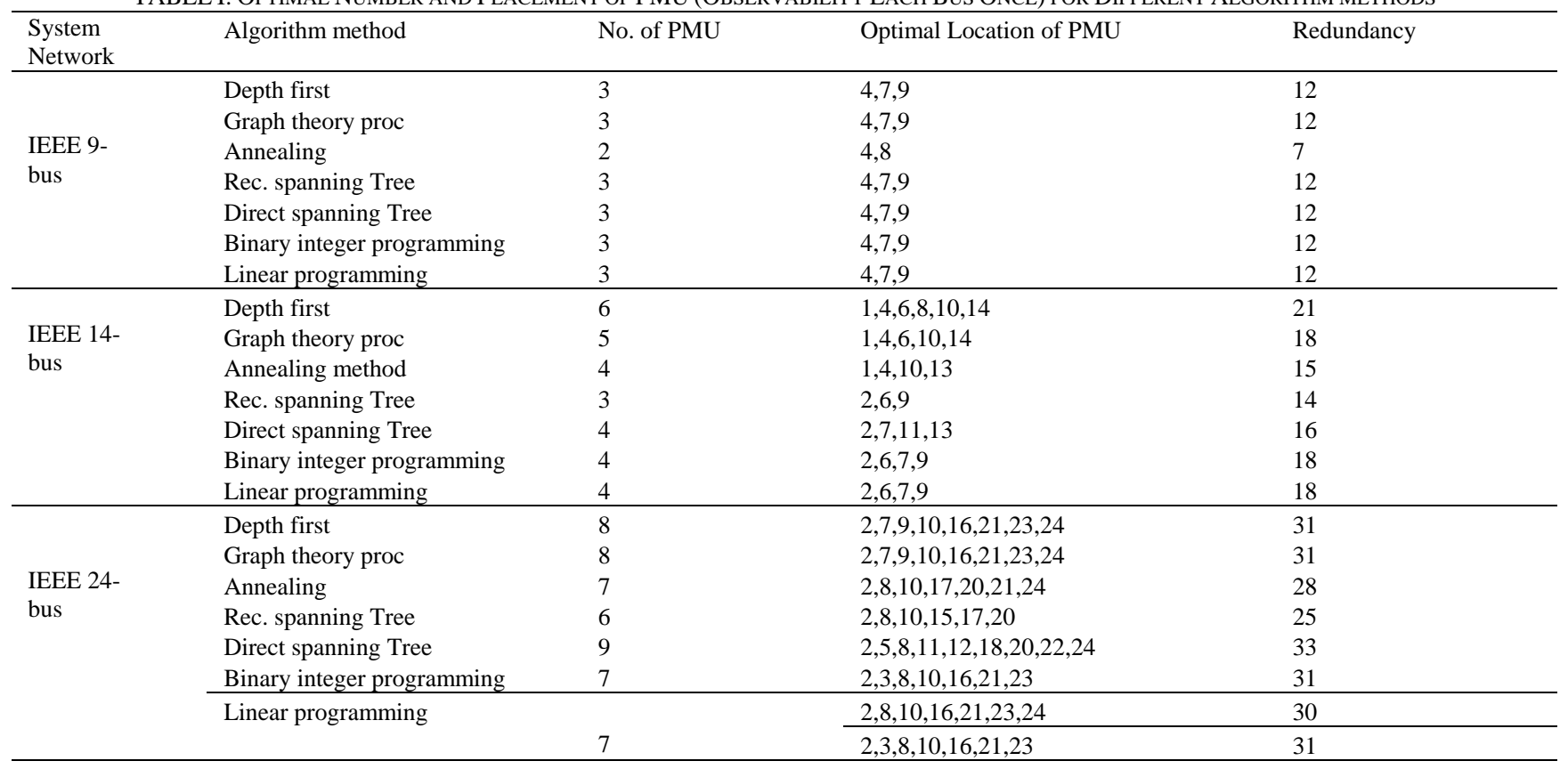

TABLE II: OPTIMAL NUMBER AND PLACEMENT OF PMU (OBSERVABILITY EACH BUSBAR TWICE) FOR DIFFERENT ALGORITHM METHODS

\begin{tabular}{|c|c|c|c|c|}
\hline $\begin{array}{l}\text { System } \\
\text { Network }\end{array}$ & Algorithm method & $\begin{array}{l}\text { No. of } \\
\text { PMU }\end{array}$ & Optimal Location of PMU & Redundancy \\
\hline IEEE 9-bus & $\begin{array}{l}\text { Dir(N-1) Spanning Tree } \\
\text { Binary integer programming } \\
\text { Linear programming }\end{array}$ & $\begin{array}{l}3 \\
6 \\
6 \\
\end{array}$ & $\begin{array}{l}4,7,9 \\
1,2,3,4,7,9 \\
1,2,3,4,7,9 \\
\end{array}$ & $\begin{array}{l}12 \\
18 \\
18 \\
\end{array}$ \\
\hline $\begin{array}{l}\text { IEEE 14- } \\
\text { bus }\end{array}$ & $\begin{array}{l}\text { Dir(N-1) Spanning Tree } \\
\text { Binary integer programming } \\
\text { Linear programming }\end{array}$ & $\begin{array}{l}8 \\
9 \\
9 \\
\end{array}$ & $\begin{array}{l}2,3,5,6,7,10,13,14 \\
2,4,5,6,7,8,9,11,13 \\
2,4,5,6,7,8,9,10,13 \\
\end{array}$ & $\begin{array}{l}32 \\
37 \\
37 \\
\end{array}$ \\
\hline \multirow[t]{3}{*}{$\begin{array}{l}\text { IEEE 24- } \\
\text { bus }\end{array}$} & $\begin{array}{l}\text { Dir(N-1) Spanning Tree } \\
\text { Binary integer programming }\end{array}$ & 14 & $\begin{array}{l}1,3,4,5,6,8,11,12,15 \\
16,17,20,21,23 \\
1,2,7,8,9,10,11,15,16 \\
17,20,21,23,24\end{array}$ & 58 \\
\hline & Linear programming & 14 & $\begin{array}{l}1,2,3,7,8,9,10,11,15 \\
16,17,20,21,23\end{array}$ & 59 \\
\hline & & & $\begin{array}{l}1,2,3,4,7,8,10,11,15 \\
16,17,20,21,23\end{array}$ & 56 \\
\hline
\end{tabular}

TABLE III: OPTIMAL NUMBER AND PLACEMENT OF PMU (OBSERVABILITY EACH BUS ONCE) FOR IRAQ NETWORK (400KV, 132KV)

\begin{tabular}{ccc}
\hline Network & Optimal No. of PMU & PMU optimal location \\
\hline Iraq 400KV & 8 & $2,6,10,12,14,16,18,23$ \\
\hline & & $1,6,11,14,15,19,20,29,30,34,35,41,49,52,56,59,64,67$, \\
Iraq 132KV & 66 & $70,74,77,82,83,86,90,95,96,100,103,108,109,113,117$, \\
& & $119,121,127,129,131,138,141,147,151,157,160,162$, \\
$165,166,172,175,177,184,188,190,194,195,200,201$, \\
\end{tabular}

\section{B. Single PMU Being Out of Used}

The proposed method is designed to maintain completely observability of system even in the case of the outage of any single PMU. In general, a bus is observed by only one PMU by using a direct or a pseudo measurement. With exception cases to double lines or more between buses, where a bus may be observed more than once by the same PMU. A minimum redundancy level of one, therefore, ensures complete system observability, in general, for any single PMU or single interconnection between bus outage. In order to increase the reliability of system observability, each bus should be observed by at least two PMUs. This ascertains that a PMU outage or interconnection between two buses outage will not lead to loss of observability. With reference to observability constraints to the system of (3), the minimum
PMU placement constraints can be formulated as follows:

$$
f_{i}=\sum_{j=1}^{n} a_{i j} x_{i} \geq 2 ? \quad i=\ldots \ldots n
$$

This relation states that all of the buses will be observable in the case of a single PMU outage or interconnection between two buses outage. Hence, constrain $f_{i} \geq 2$ makes the system be observable by two PMUs [7].

To illustrate this case take the example above of 7-bus system of Fig. 1 is used. And rewrite Eq. (8) to be: the observability constraints in (9) ,The operator "+" serves as the logical "OR" and the use of 2 in the right hand side of the inequality constraints ensures that at least two of the variables appearing in the sum will be non-zero. The constraint $f_{1} \geq 2$ implies that PMU must be placed at both 
buses 1 and 2 in order to make bus 1 observable twice. the second observability constraint $f_{2} \geq 2$ indicates that at least two PMU should be installed at any two of the buses 1, 2, 3, 6, or 7 in order to make bus 2 observable twice, and same away for others observability constraints. The zero injection buses and conventional measurements don't take in this paper to observability of critical part of grid.

$$
f(x)=\left\{\begin{array}{lc}
f_{1}=x_{1}+x_{2} & \geq 2 \\
f_{2}=x_{1}+x_{2}+x_{3}+x_{6}+x_{7} & \geq 2 \\
f_{3}=x_{2}+x_{3}+x_{4}+x_{6} & \geq 2 \\
f_{4}=x_{3}+x_{4}+x_{5}+x_{7} ? & \geq 2 \\
f_{5}=x_{4}+x_{5} & \geq 2 \\
f_{6}=x_{2}+x_{3}+x_{6} & \geq 2 ? \\
f_{7}=x_{2}+x_{4}+x_{7} & \geq 2
\end{array} ?\right.
$$

\section{Test REsUlts AND Discussion}

There are two cases study tested for networks of IEEE 9-bus, IEEE 14-bus, and IEEE 24-bus systems with different algorithms to determine optimal numbers and optimal placement of PMU.

\section{A. Observability of Each Bus with Single PMU}

In this case every bus bar can be seen at least one time from PMU by direct or indirect measurement. To find the optimal numbers and optimal placement of PMU to systems using PSAT toolbox with different methods; Depth First method, Annealing method, direct spanning and Graph Theoretic Procedure, and MATLAB optimization with linear programming and binary integer programming.

The strategic choice of the minimum number of PMUs and the optimal location of the PMUs in order to ensure complete observability using the objective function in (2) to determined minimum number of PMU depending to Constraints in (3).

Which determined placement of the PMU depending to maximum branches of bus bar in these constraints and then satisfy maximum redundancy in (5).

Whatever method is used to PMU placement, it can be obtained by put it at bus bar at maximum branches then check observability of the system If the system is observable, then the placement stops, Otherwise the placement must be continued.

Table I shows the test results of proposed methods, there is more than one solution to determine optimal numbers and optimal placement of PMUs set considering no zero injection buses.

The simulation results shown in Table I for IEEE 9-bus system can be illustrated in Fig. 2(a) each bus can be seen at least by one PMU in the optimization methods depth first, graph theory procedure, Rec. spanning Tree, direct spanning Tree, linear programming, and binary integer programming with 3 PMU to Observability of whole system and 2 PMU use at annealing methods but the buses $\{2,3\}$ it be unobservability.

the simulation results shown in Table I for IEEE 14-bus system can be illustrated in Fig. 2(b) Each bus can be seen at least by one PMU in the optimization methods, depth first with 6 PMU to Observability of whole system, graph theory procedure with 5 PMU to observability of whole system but the bus $\{8\}$ it be unobservability, annealing with 4 PMU to observability of whole but the bus $\{8\}$ it be unobservability, Rec. spanning Tree with 4 PMU to Observability of whole system but the bus $\{8\}$ it be unobservability, direct spanning Tree with 4 PMU to observability of whole system, and linear programming and binary integer programming with 4 PMU to Observability of whole system.

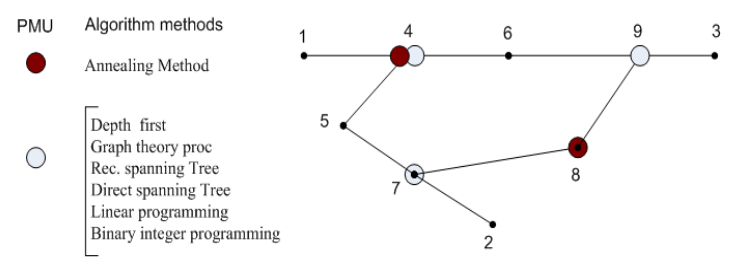

(a)

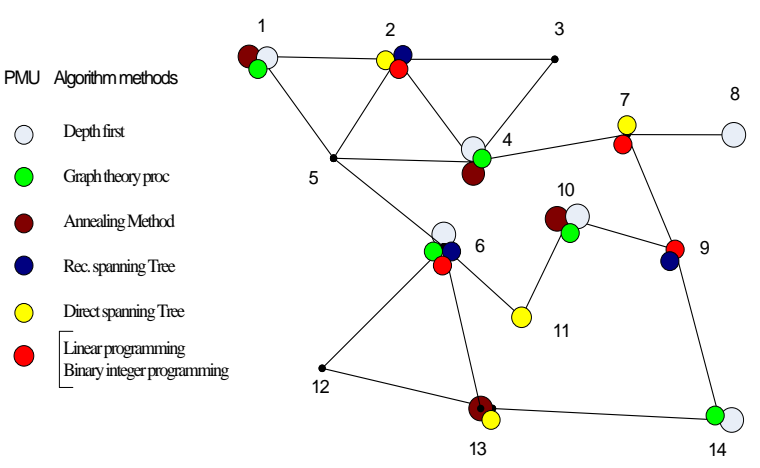

(b)

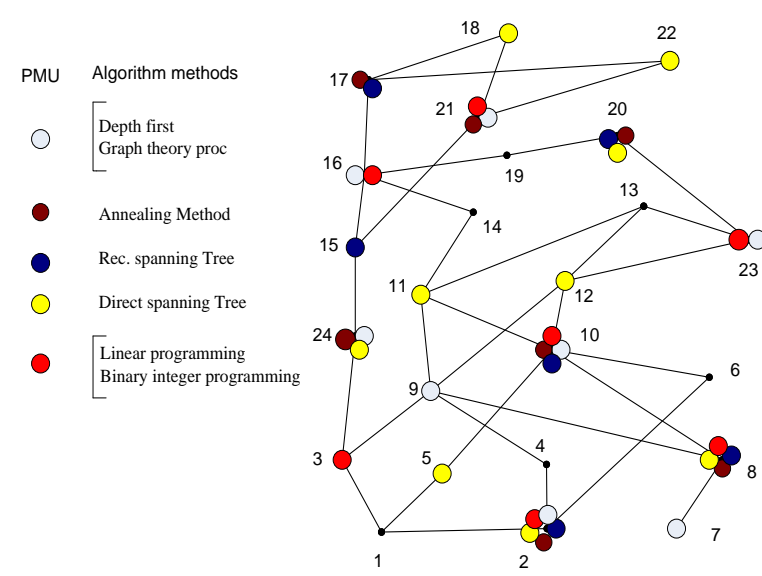

(c)

Fig. 2. Optimal placement of PMU (observability each bus once) for different algorithm method to; (a) IEEE 9-bus system; (b) IEEE 14-bus system; (c) IEEE 24-bus system.

The simulation results shown in Table I for IEEE 24-bus system can be illustrated in Fig. 2(c) each bus can be seen at least by one PMU in the optimization methods, depth first and graph theory procedure with 8 PMU to observability of whole system, annealing method with 7 PMU use observability of whole system but the buses $\{13,14\}$ it be unobservability, Rec. spanning Tree with 6 PMU to Observability of whole system but the buses $\{3,13,14\}$ it be unobservability, direct spanning Tree with 9 PMU to observability of whole system , and linear programming, binary integer programming with 7 PMU to observability of whole system. 
TABLE IV: OPTIMAL NUMBER AND PLACEMENT OF PMU (OBSERVABILITY EACH BUS TWICE) FOR IRAQ NETWORKS (400KV, 132KV)

\begin{tabular}{ccc}
\hline Network & Optimal No. of PMU & PMU OPTIMAL LOCATION \\
\hline Iraq 400KV & 17 & $1,2,4,5,6,9,10,12,13,14,15,16,18,20,23,24,25$ \\
\hline & $2,3,5,6,7,8,11,13,14,15,16,18,19,21,24,25,26,27,29$, \\
& & $30,31,32,33,34,35,37,38,39,40,41,42,43,45,46,47,49$, \\
Iraq 132KV & $50,52,53,55,56,58,59,60,61,64,65,67,69,70,72,74,75$, \\
& $76,77,79,80,82,83,84,86,87,89,90,91,92,93,94,95,96$, \\
& $97,98,100,101,102,103,106,107,108,109,110,113,114$, \\
& $115,116,117,119,120,121,123,125,126,127,129,130$, \\
& $131,132,133,136,138,139,140,143,144,146,147,151$, \\
& $152,154,158,160,162,163,164,165,166,168,169,171$, \\
& $172,173,174,175,177,178,180,181,182,184,187,188$, \\
& $190,191,193,194,195,197,200,201,203,204,206,207$, \\
\end{tabular}

\section{B. Observability of Each Bus with two PMU}

In this case every bus bar can be seen at least two times from PMU by direct or indirect measurement. To find the optimal numbers and optimal placement of PMU to systems using PSAT program with Dir (N-1) Spanning Tree and MATLAB optimization with linear programming and binary integer programming.

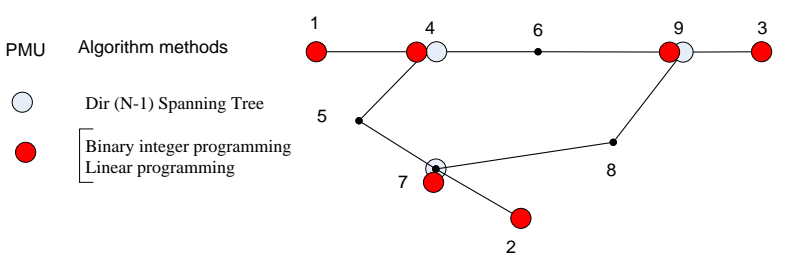

(a)

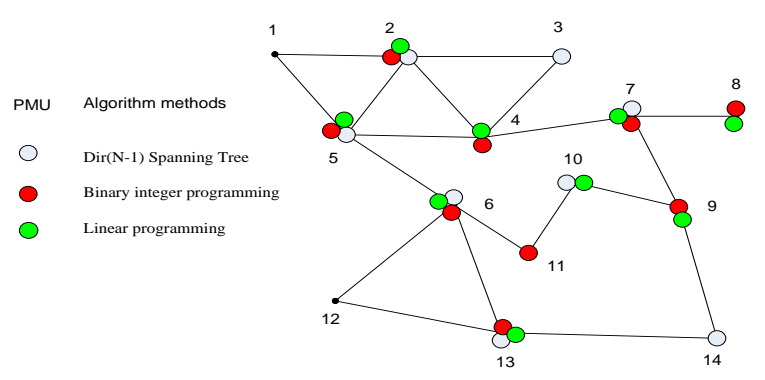

(b)

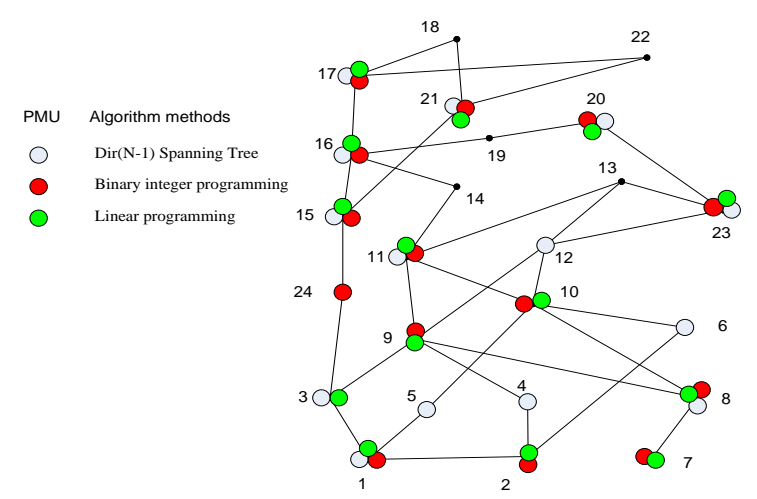

(c)

Fig. 3. Optimal placement of PMU (observability each bus twice) for different algorithm method to (a) IEEE 9-bus system (b) IEEE 14-bus system(c) IEEE 24-bus system

The strategic choice of the minimum number of PMUs and the optimal location of the PMUs in order to ensure complete observability using the objective function in (2) to determined minimum number of PMU depending to constraints in (8) which determined placement of the PMU depending to maximum branches of bus bar in these constraints and then satisfy maximum redundancy in (5).

Whatever method is used to PMU placement, it can be obtained by put it at busbar at maximum branches then check observability of the system If the basbars of system is observable twice, then the placement stops, Otherwise the placement must be continued.

Table II shows the test results of proposed methods, there is more than one solution to determine optimal numbers and optimal placement of PMUs set considering no zero injection buses.

The simulation results shown in Table II for IEEE 9-bus system can be illustrated in Fig. 3(a) each bus can be seen at least by two PMU in the optimization methods Dir (N-1) Spanning Tree with 3 PMU to observability of whole system but the buses $\{1,2,3,4,7,9\}$ it be observability for only one time, linear programming, and binary integer programming with 6 PMU to Observability of whole system at least two times.

The simulation results shown in Table II for IEEE 14-bus system can be illustrated in Fig. 3(b) each bus can be seen at least by two PMU in the optimization methods Dir (N-1) Spanning Tree with 8 PMU to Observability of whole system but the bus $\{8\}$ it be observability for only one time, linear programming, and binary integer programming with 9 PMU to Observability of whole system at least two times.

The simulation results shown in Table II for IEEE 24-bus system can be illustrated in Fig. 3(c) each bus can be seen at least by two PMU in the optimization methods Dir (N-1) Spanning Tree with 14 PMUs to Observability of whole system but the bus $\{4,6,7,8,11\}$ it be observability for only one time, linear programming, and binary integer programming with 14 PMUs to Observability of whole system at least two times.

It is clear from analysis of result for two cases above to get whole system observability with maximum redundancy it can be obtained by used binary integer programming or linear programming but in case linear programming its give more from one solution.

Therefore, Utilized the binary integer programming for determined the optimal number and optimal placemen of PMUs to Iraq networks (400KV, 132KV). 


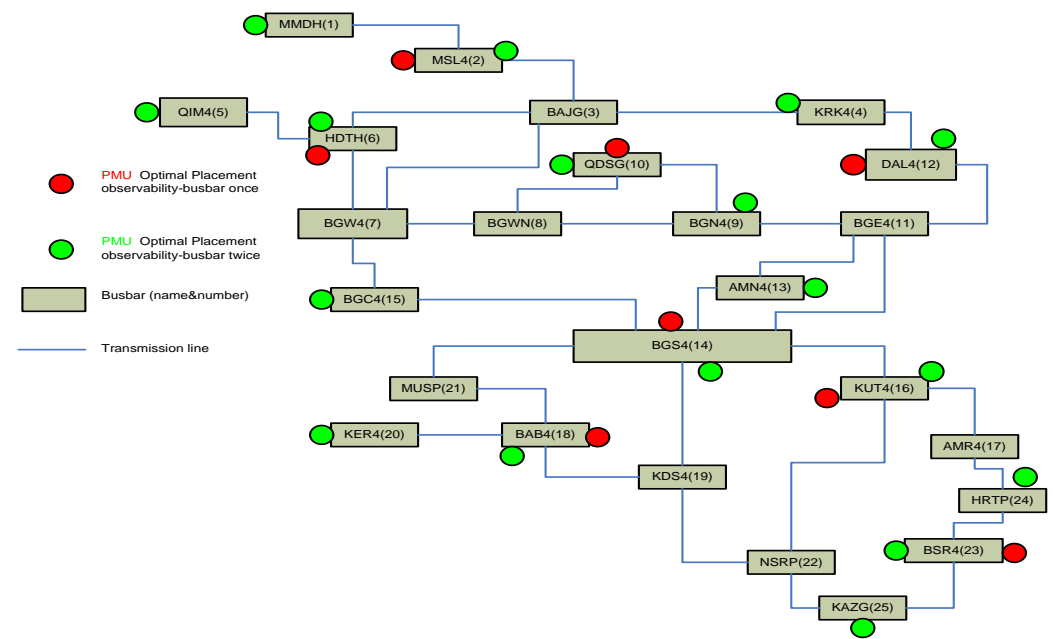

Fig. 4. Single line diagram of the Iraq network $400 \mathrm{KV}$ with optimal placement of PMU.

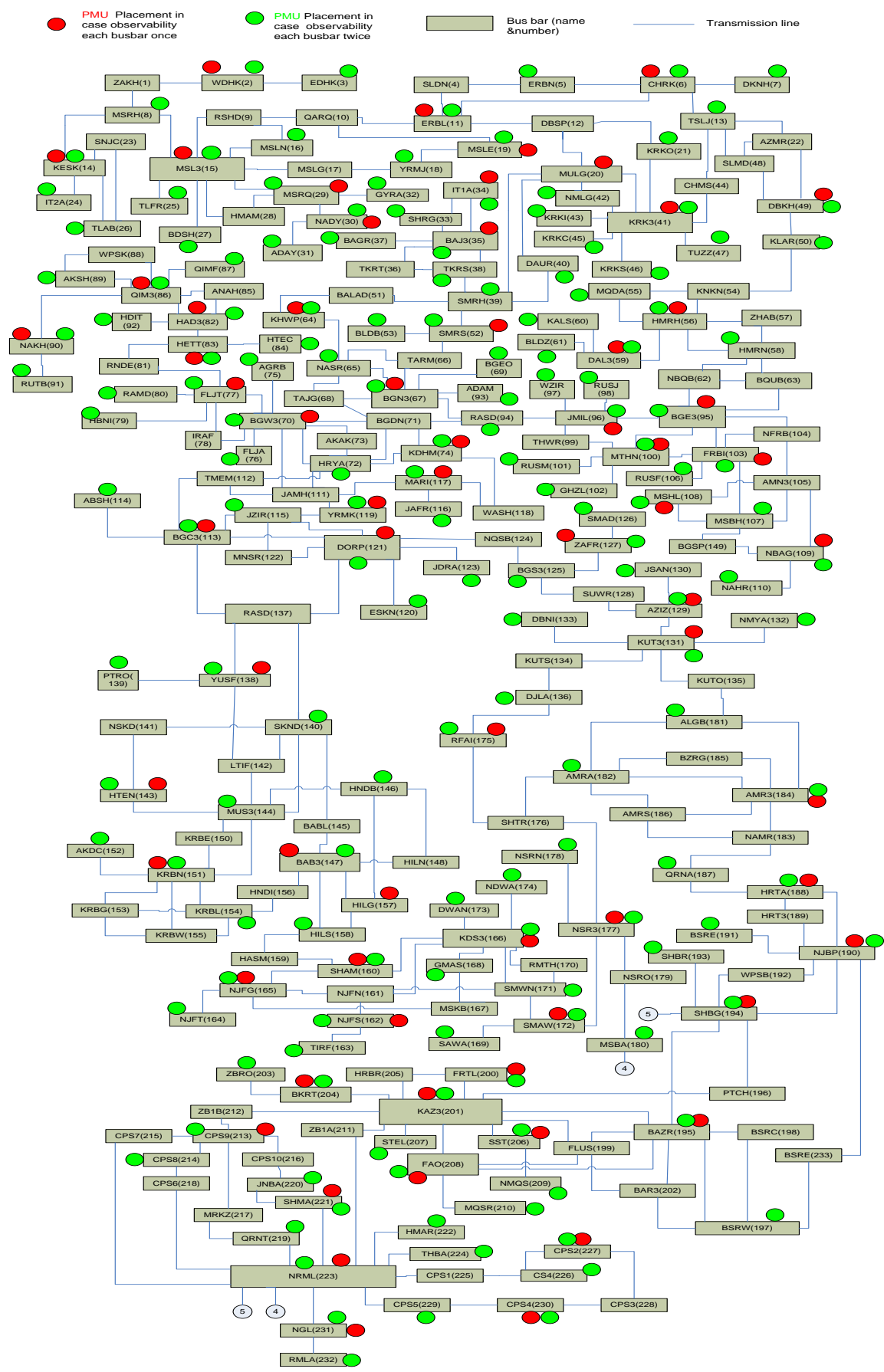

Fig. 5. Single line diagram of the Iraq network $132 \mathrm{KV}$ with optimal placement of PMU. 
The Iraq generation and transmission network comprises 25 bus bars $400 \mathrm{KV}$, and 233 bus bars $132 \mathrm{KV}$ distributed at different situation of country (Iraq Power Grid 400KV and 132KV Overall Single Line Diagram), the map of Iraq power grid represented In the form of busbars names and the numbers of busbars randomly therefore, Has been re-arranged the map in the form blocks represent busbar name and busbar number and interconnection between these blocks(basbars) by lines represent transmission lines.

The transmission lines between busbars represented here only as single transmission line between busbars as shown in Fig.4, Fig.5 respectively Because it is costly to put all busbar with PMU, and efficient of binary integer programming to obtain optimal number and optimal placement of PMU to observability of whole system, for the two cases mentioned above As shown in simulation results in Tables 3, 4 and Fig.4, and Fig. 5.

The simulation results shown in Table III for Iraq networks $(400 \mathrm{KV}, 132 \mathrm{KV})$ each bus can be seen at least by one PMU in the optimization method, binary integer programming with 8 PMU to Observability of whole system of $400 \mathrm{KV}$ network as shown in Fig.4, and 66 PMU to Observability of whole system of $132 \mathrm{KV}$ network as shown in Fig. 5.

The possibility of single PMU out of used it can be happened for many reasons such as do routine maintenance or loss communication channel... etc. for the systems to be reliability and flexibility so each bus should be seen at least by two PMU. the simulation results shown in Table IV for Iraq networks $(400 \mathrm{KV}, 132 \mathrm{KV})$ system each bus bar can be seen at least by two PMU in the optimization method, binary integer programming with 17 PMU to observability of whole system of 400KV network as shown in Fig.4, and 160 PMU to observability of whole system of $132 \mathrm{KV}$ network as shown in Fig.5 Its clear from result its required PMU at bus bars less than the numbers of total bus bars, but also its look high numbers because there are many terminal substation at network systems.

\section{CONCLUSION}

Principles of power system Observability with PMU placements it is offered through this paper. the cost minimization of the installation of PMUs in power system by minimizing the number of PMUs for observability of whole system. Besides the placement of PMUs, multiple choices of locations are eliminated by selecting the combination of buses having maximum redundancy, the possibility of the losing of one of PMUs in system it is taken into account. Simulation results on IEEE-9 bus, IEEE-14bus and IEEE-24 bus test systems with Different optimization methods indicate that binary integer programming amongst others methods satisfactorily provides full observable whole system measurements with maximum redundancy and minimum number of PMUs. the optimal number and optimal location of PMUs to the Iraq networks $(400 \mathrm{KV}, 132 \mathrm{KV})$ to obtain observability of whole system in two cases: first case each bus can be seen at least one time from PMU and second case each bus can be seen at least two times from PMU, has been investigated by using binary integer programming. Simulation results for different networks showed efficiency and effectiveness of binary integer programming to obtaining the minimum number and optimal locations of PMUs required for complete observability of network.

\section{ACKNOWLEDGEMENTS}

We thank everyone at the HUST/department of Electrical \& Electronic Engineering who participated in this research for many stimulating discussions. Also special thanks Ministry of electricity of Iraq/Training \& Development office and Energy Transmission of Euphrates Middle region.

\section{REFERENCES}

[1] Q. K. Mohsin, X. N. Lin, Z. C. Wang, O. Sunday, M. S. Khalid, and P. W. Zheng, "Iraq network $400 \mathrm{kV}, 50 \mathrm{~Hz}$ interconnect with Iran, Turkey and Syria using phase-shifting transformers in control and limit power flow of countries," in Proc. IEEE Power and Energy Engineering Conference, 2014.

[2] D. Ghosh, T. Ghose, and D. K. Mohanta, "Reliability analysis of a geographic information system-aided optimal phasor measurement unit location for smart grid operation," in Proc IMechE Part O: J Risk and Reliability, vol. 227, no. 4, pp. 450-458, 2013

[3] V. Khiabani, R. Kavesseri, and K. Farahmand, "A reliability based multi-objective formulation for optimal PMU placement," International Review on Modelling and Simulations, vol. 5, no. 4, 2012.

[4] G. Venugopal, R. Veilumuthu, and P. A. Theresa, "Optimal PMU placement and observability of power system using PSAT," International Journal of Computer Networks and Security, October, 2013.

[5] X. Tai, D. Marelli, E. Rohr, and M. Y. Fu, "Optimal PMU placement for power system state estimation with random component outages," International Journal of Electrical Power and Energy Systems, vol. 51, pp. 35-42, April, 2013.

[6] M. Panteli, P. A. Crossley, D. S. Kirschen, and D. J. Sobajic, "Assessing the impact of insufficient situation awareness on power system operation," IEEE Transactions on Power Systems, vol. 28, no. 3, pp. 2967- 2977, August, 2013.

[7] T. T. Cai and Q. Ai, "Research of PMU optimal placement in power systems," in Proc. the 5th Wseas/Iasme Int. Conf. on systems theory and Scientific Computation, Malta, September, pp. 38-43. 2005.

[8] V. Khiabani, and K. Farahmand, "Max covering phasor measurement units placement for partial power system observabilitity," Engineering Management Research, vol. 2, no. 1, pp. 43-54, March 4, 2013.

[9] B. Xu and A. Abur, "Observability analysis and measurement placement for systems with PMUs," in Proc. the IEEE PES Power Systems Conference and Exposition, vol. 2, pp. 943-946, 2004.

[10] B. Xu and A. Abur, "Optimal placement of phasor measurement units for state estimation," Final Project Report, PSERC 2005.

[11] J. Peng, Y. Sun, and H. F. Wang, "Optimal PMU placement for full network observability using Tabu search algorithm," Int J Electr Power Energy System, 2006.

[12] X. Bei, Y. J. Yoon, and A. Abur, "Optimal placement and utilization of phasor measurements for state estimation," in Proc. 15th Power Systems Computation Conference, 2005.

[13] K. Jamuna and K. S. Swarup, "Optimal placement of PMU and SCADA measurements for security constrained state estimation," International Journal Electrical Power Energy System, vol. 33, no. 10, pp. 1658-1665, 2011.

[14] A. Ahmadi, Y. A. Beromi, and M. Moradi, "Optimal PMU placement for power system observability using binary particle swarm optimization considering measurement redundancy," Expert System Apply Sci Direct, vol. 38, no. 6, pp. 7263-9, 2011.

[15] V. Khiabani, E. Erdem, K. Farahmand, and K. Nygard, "Genetic algorithm for instrument placement in smart grid," Nature and Biologically Inspired Computing (NaBIC), pp. 214-219, 2013.

[16] V. Khiabani, E. Erdem, K. Farahmand, and K. Nygard, "smart grid pmu allocation using genetic algorithm," Journal of Network and Innovative Computing, vol. 2, no. 1, pp. 30-40, 2014.

[17] B. Allagui, I. Marouani, and H. H. Abdallah, "Optimal placement of phasor measurement units by genetic algorithm," International Journal of Energy and Power Engineering, vol. 2, no. 1, pp. 12-17, February, 2013. 
[18] B. K. S. Roy, A. K. Sinha, and A. K. Pradhan, "An optimal PMU placement technique for power system observability," Electrical Power and Energy Systems, vol. 42, pp. 71-77, May, 2012.

[19] L. Huang, Y. Z. Sun, J. Xu, W. Z. Gao, J. Zhang, and Z. P. Wu, "Optimal PMU placement considering controlled islanding of power system," IEEE Transactions on Power Systems, vol. 29, no. 2, 2014.

[20] K. Mazlumi, M. Azari, and S. Beheshti, "Optimal multistage scheduling Of PMU placement for power system observability," International Journal on Technical and Physical Problems of Engineering, vol. 4, no. 4, Dec. 2012.

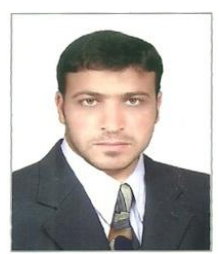

Qasim Kamil Mohsin received his master's degree (M. Tech) in electrical and electronics engineering from Sam Higginbottom Institute of Agriculture, Technology \& Sciences, Allahabad, India in 2013 $\mathrm{He}$ is currently perusing his $\mathrm{PhD}$ degree in electrical engineering from Huazhong University of Science and Technology, Wuhan, China. His research interests include power system operation, protection and control, and renewable energy. In his country, he is affiliated with ministry of electricity of Iraq, directorate general of energy transmission of Euphrates Middle Region. He works in high voltage networks for protective relays testing and settings, Maintenance of the electrical equipments (circuit breakers, transformers, current transformers and voltage transformers)

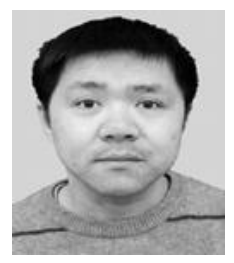

Xiangning Lin received the M.S. and Ph.D. degrees in School of Electrical and Electronic Engineering of Huazhong University of Science and Technology (HUST), Wuhan, China. Currently, he is a professor at HUST. His research interests are modern signal processing and its application in power systems as well as power system protective relaying and control.

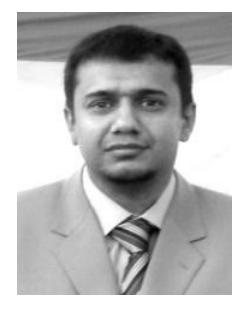

Asad Waqar received his master's degree in electrical power engineering from RWTH Aachen, Germany in 2011. He is currently perusing the $\mathrm{PhD}$ degree from Huazhong University of Science and Technology, Wuhan, China. His research interests include grid integration of renewable energy, microgrid planning under uncertainty, power system operation and control. He is also a faculty member in the Department of Electrical Engineering at COMSATS Institute of Information Technology, Pakistan.

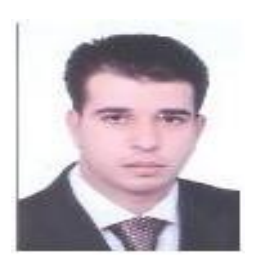

Samir Mohammed received the M.Sc. degree from Tanta University, at the Faculty of Engineering, Tanta, Egypt, in 2012, He is currently perusing his $\mathrm{PhD}$ degree in electrical engineering from Huazhong University of Science and Technology, Wuhan, China. His research interests feasibility study of hybrid Micro grids optimization schemes. $\mathrm{He}$ is currently an assistant lecturer on Tanta University, Faculty of Engineering, Tanta University, Egypt. 